\title{
Efecto del extracto de Allium sativum, Citrus limon y Carica papaya en parasitosis intestinal
}

\author{
Effect of Allium sativum, Citrus limon and Carica papaya extract on intestinal \\ parasitosis
}

Juana Irma Palacios Zevallos 1,a, Julia Marina Palacios Zevallos 1,a, Nancy Doris, Calzada Gonzales 2,a a

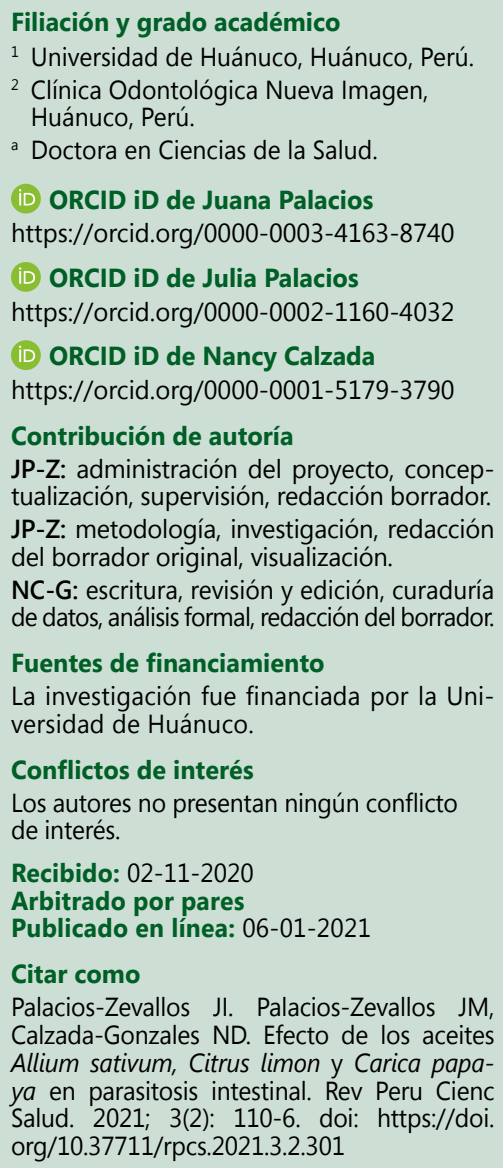

\section{RESUMEN}

Objetivo. Evaluar el efecto del extracto de Allium sativum (ajo), Citrus limon (limón) y Carica papaya (papaya) en el tratamiento de parasitosis intestinal. Métodos. Estudio experimental, prospectivo, transversal. El tamaño de la muestra se calculó por muestreo no probabilístico intencionado. Para evaluar el efecto del extracto, primero se llevó a cabo la elaboración del extracto experimental antiparasitario a base de Allium sativum (ajo), Citrus limon (limón) y Carica papaya (papaya); se administró cada 12 horas durante cinco días; y se recolectó la muestra (heces) para el análisis parasitológico. Las muestras para el análisis de los datos se aplicaron la prueba no paramétrica de McNemar y la prueba exacta de Fisher. Resultados. El análisis estadístico mostró diferencia significativa $(p<0,05)$ entre antes y después de administrar el extracto experimental antiparasitario, tanto en los protozoarios Giardia lamblia y helmintos Tenia solium. Con relación a los tres grupos etarios de 6 a 7 años, 8 a 9 años y 10 a 12 años; no hubo diferencia estadísticamente significativa en el efecto antiparasitario $(p>0,05)$. Conclusiones. El extracto experimental Allium sativum (ajo), Citrus limon (limón) y Carica papaya (papaya) presenta diferencias significativas después ser administrado frente a la parasitosis intestinal.

Palabras clave: Allium sativum; Citrus; Carica; parasitosis intestinales, protozoario; helminto (Fuente: DeCS-BIREME).

\section{ABSTRACT}

Objective. To evaluate the effect of Allium sativum (garlic), Citrus limon (lemon) and Carica papaya (papaya) extracts in the treatment of intestinal parasitosis. Methods. Experimental, prospective, cross-sectional study. The sample size was calculated by non-probabilistic purposive sampling. To evaluate the effect of the extract, the experimental antiparasitic extract based on Allium sativum (garlic), Citrus limon (lemon) and Carica papaya (papaya) was first developed; it was administered every 12 hours for five days; and the sample (feces) was collected for parasitological analysis. The samples for data analysis were subjected to the McNemar nonparametric test and Fisher's exact test. Results. The statistical analysis showed a significant difference $(p<$ 0.05 ) between before and after administering the experimental antiparasitic extract, both in protozoa (Giardia lamblia) and helminths (Taenia solium). In relation to the three age groups (6 to 7 years, 8 to 9 years and 10 to 12 years), there was no statistically significant difference in the antiparasitic effect ( $p>0.05$ ). Conclusions. The experimental extract (Allium sativum (garlic), Citrus limon (lemon) and Carica papaya (papaya) shows significant differences after being administered against intestinal parasitosis.

Keywords: Allium sativum; Citrus; Carica; intestinal parasitosis, protozoan; helminth (Source: MeSH - NLM). 


\section{INTRODUCCIÓN}

La parasitosis intestinal está considerada como un problema de salud pública a nivel mundial, estimándose en más de un cuarto de la población del mundo con parásitos, siendo la población infantil la más afectada ${ }^{(1-3)}$. El Perú, en vías de desarrollo se ha podido encontrar una prevalencia alta de parásitos, lo cual demuestra que nuestro país no es ajeno a esta enfermedad. De acuerdo a las regiones del Perú, prevalecen diferentes parásitos ${ }^{(4-6)}$.

Estudios sobre el Allium sativum (ajo) revelan los beneficios medicinales y las propiedades terapéuticas del ajo, los cuales se atribuyen a la presencia de compuestos orgánicos de azufre ${ }^{(7,8)}$. Omayma et al. han demostrado que los componentes de la fruta tropical Carica papaya (papaya) tiene importantes propiedades anticestodales que permiten que su extracto de semilla sea una alternativa muy efectiva al praziqueantel contra Hymenolepsis nana ${ }^{(9)}$, toda vez que el isotiocianato de bencilo es el ingrediente antihelmíntico bioactivo en las semillas de papaya ${ }^{(10)}$.

La infección por protozoos intestinales patógenos y helmintos provoca un deterioro de la calidad de vida, desnutrición, pérdida de apetito, problemas cognitivos y mortalidad considerables en todo el mundo, especialmente entre los niños pequeños de los países en desarrollo y las personas inmunodeprimidas ${ }^{(11)}$. Por lo expuesto anteriormente el estudio tuvo como objetivo evaluar el efecto del aceite de Allium sativum (ajo), Citrus limon (limón) y Carica Papaya (papaya) en el tratamiento de parasitosis intestinal.

\section{MÉTODOS}

\section{Tipo de estudio}

Se realizó un estudio clínico analítico, diseño cuasiexperimental, de enfoque cuantitativo y en el área de estudio fitoterapia en medicina.

\section{Población y muestra}

La población estuvo constituida por todos los escolares de 6 a 12 años del centro poblado Jancao (Huánuco), entre agosto y octubre del 2018. Se utilizó el muestreo no probabilístico intencionado; la muestra total fue de 40 sujetos con diagnóstico de parasitosis intestinal causada por Giardia lamblia, Tenia solium o ambos parásitos, que recibieron el extracto experimental antiparasitario Allium Sativum (ajo), Citrus Limon (limón) y Carica Papaya (papaya). De estos, 23 sujetos dieron positivo para protozoario más helminto, 10 para protozoario
(Giardia lamblia) y 7 para helmintos (Tenia solium). Los criterios de inclusión fueron: sujetos de ambos sexos de 6 a 12 años, que hayan firmado los apoderados el consentimiento informado y con diagnóstico de parasitosis infantil causado por Giardia lamblia, Tenia solium o ambos parásitos. Los criterios de exclusión fueron: hipersensibilidad previa a productos de papaya, ajos o limón o haber recibido algún antihelmíntico en las dos semanas anteriores a la inscripción y durante el estudio.

\section{Instrumentos de recolección de datos}

El instrumento utilizado para medir fue la ficha de observación, la cual fue validada mediante juicio de expertos por cinco profesionales que evaluaron la claridad en la redacción, consistencia y coherencia. En la primera sección se consideró los datos sociodemográficos de los sujetos de estudio edad, sexo y procedencia. En el segundo apartado las características parasitológicas: presencia o ausencia de parasitosis intestinal al inicio y a los 5 días de administrada la esencia experimental, tipo de parásito (protozoario o helmintos), al inicio y a los 5 días de administrada la esencia experimental. La dosis de administración fue cada 12 horas.

\section{Procedimientos de la recolección de datos}

1. Cuarenta fueron los sujetos que cumplieron con los criterios de inclusión y exclusión, para ser suministrado el extracto experimental antiparasitario: Allium sativum (ajo), Citrus limon (limón) y Carica papaya (papaya).

2. La preparación del extracto se realizó de la siguiente manera: el ajo fresco, papaya y limón fresco fueron comprados en el mercado modelo de la ciudad de Huánuco. Los bulbos de ajo se separaron, se pelaron y se lavaron con agua destilada, también la flor de papaya se lavó con agua destilada. Después de secar en un esterilizador ambos productos, se trituraron $500 \mathrm{~g}$ de bulbos de ajo y flor de papaya limpios y el zumo de limón, usando una licuadora comercial (Oster, Estados Unidos). El extracto resultante se diluyó con agua destilada ( $1 \mathrm{~g} / \mathrm{mL}$ ) para preparar una solución acuosa. La solución se filtró correctamente. El extracto experimental se colocó en tubos de 1,5 ml y se almacenó en un congelador a $-20^{\circ} \mathrm{C}$. La dosis seleccionada para el presente estudio $(50 \mathrm{mg} / \mathrm{kg}$ de peso corporal) cada 12 horas por cinco días, corresponde a la cantidad diaria de ajo recomendada para el consumo humano $(4 \mathrm{mg})^{(17)}$. El extracto de ajo, flor de papaya y limón se administró a los sujetos de estudio. Las sustancias se almacenaron a $4{ }^{\circ} \mathrm{C}$ y se recuperaron solo durante su uso. 
Tabla 1. Comparación de parasitosis intestinal (Giardia lamblia + Tenia solium), antes y después de administrar extracto experimental

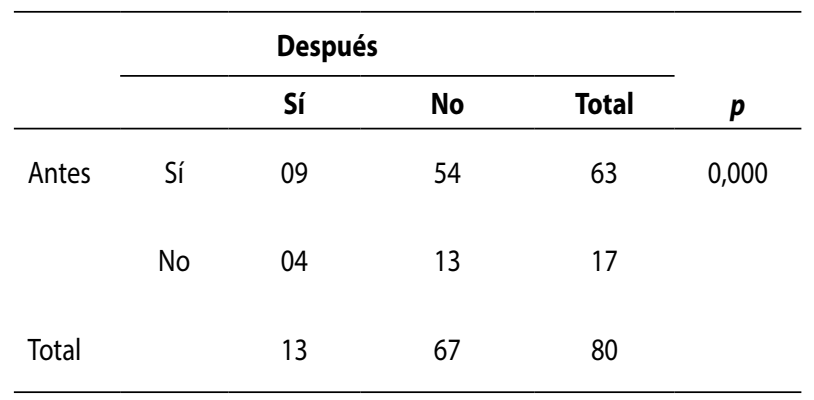

Nota. McNemar's $x^{2}=26,00$

\section{Análisis de datos}

Para el análisis descriptivo e inferencial de datos se utilizó el programa estadístico STATA v.16. Para comparar los dos grupos de estudios o muestras independientes se utilizó la prueba no paramétrica MacNemar y la prueba chi-cuadrado de Pearson, debido a que los datos no cumplen con los supuestos.

\section{Aspectos éticos}

Antes de formar parte del estudio, los padres/tutores de los escolares del centro educativo recibieron una explicación completa del mismo, incluidos los riesgos y beneficios involucrados, y se obtuvo el consentimiento y asentimiento informado por escrito. El estudio se realizó siguiendo los principios éticos de la investigación médica en seres humanos según la Declaración de Helsinki de la Asociación Médica Mundial. Se obtuvo la aprobación del Comité de Ética de la Universidad de Huánuco.

\section{RESULTADOS}

En los resultados de las muestras de heces de los 40 sujetos estudiados, se encontró 23 de ellos con diagnóstico de parasitosis intestinal por protozoario (Giardia lamblia) más helminto (Tenia solium), en 10 sujetos solo protozoario (Giardia lamblia) y en 7 dio positivo para helminto (Tenia solium), haciendo un total de 33 casos para parasitosis

Tabla 2. Comparación de parasitosis intestinal (Giardia lamblia), antes y después de administrar extracto experimental

\begin{tabular}{|c|c|c|c|c|c|}
\hline & \multicolumn{3}{|c|}{ Después } & \multirow[b]{2}{*}{ Total } & \multirow[b]{2}{*}{$p$} \\
\hline & & Sí & No & & \\
\hline \multirow[t]{2}{*}{ Antes } & Sí & 07 & 26 & 33 & 0,000 \\
\hline & No & 00 & 07 & 07 & \\
\hline Total & & 07 & 33 & 40 & \\
\hline
\end{tabular}

Nota. McNemar's $x^{2}=26,00$

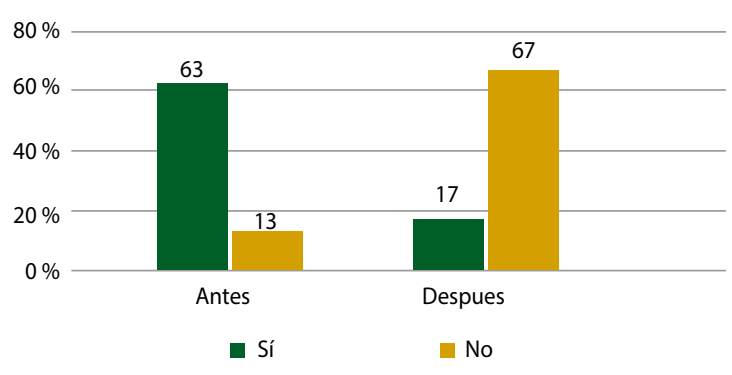

Figura 1. Representación gráfica de la comparación de parasitosis intestinal (Giardia lamblia + Tenia solium) antes y después de administrar extracto experimental

intestinal por protozoario y 30 casos para parasitosis intestinal por helminto.

En la tabla 1 y figura 1 se presentan la comparación de parasitosis intestinal por protozoario (Giardia lamblia + Tenia solium), antes y después de administrar extracto experimental Allium sativum (ajo), Citrus limon (limón) y Carica papaya (papaya). Antes de administrar se encontraron 63 casos y después de la exposición al extracto se encontraron 13 casos de parasitosis intestinal. Por lo tanto, hubo diferencia estadísticamente significativa en el efecto antiparasitario $(p<0,05)$.

En la tabla 2, se presenta la comparación de parasitosis intestinal por protozoario (Giardia lamblia), antes y después de administrar extracto experimental Allium sativum (ajo), Citrus limon (limón) y Carica papaya (papaya). Antes de administrar se encontró 33 casos y después de la exposición al extracto se encontró 7 casos de parasitosis intestinal. Por lo tanto, hubo diferencia estadísticamente significativa en el efecto antiparasitario $(p<0,05)$.

En la tabla 3, se presenta la comparación de parasitosis intestinal por helminto (Tenia solium), antes y después de administrar extracto experimental Allium sativum (ajo), Citrus limon (limón) y Carica papaya (papaya). Antes de administrar se encontraron 30 casos y después de la

Tabla 3. Comparación de parasitosis intestinal (Tenia solium), antes y después de administrar extracto experimental

\begin{tabular}{|c|c|c|c|c|c|}
\hline & \multicolumn{3}{|c|}{ Después } & \multirow[b]{2}{*}{ Total } & \multirow[b]{2}{*}{$p$} \\
\hline & & Sí & No & & \\
\hline \multirow[t]{2}{*}{ Antes } & Sí & 02 & 28 & 30 & 0,000 \\
\hline & No & 04 & 06 & 10 & \\
\hline Total & & 6 & 34 & 40 & \\
\hline
\end{tabular}

Nota. McNemar's $X^{2}=18,00$ 
Tabla 4. Parasitosis intestinal (Giardia lamblia + Tenia solium) antes y después de administrar extracto experimental según grupo etario

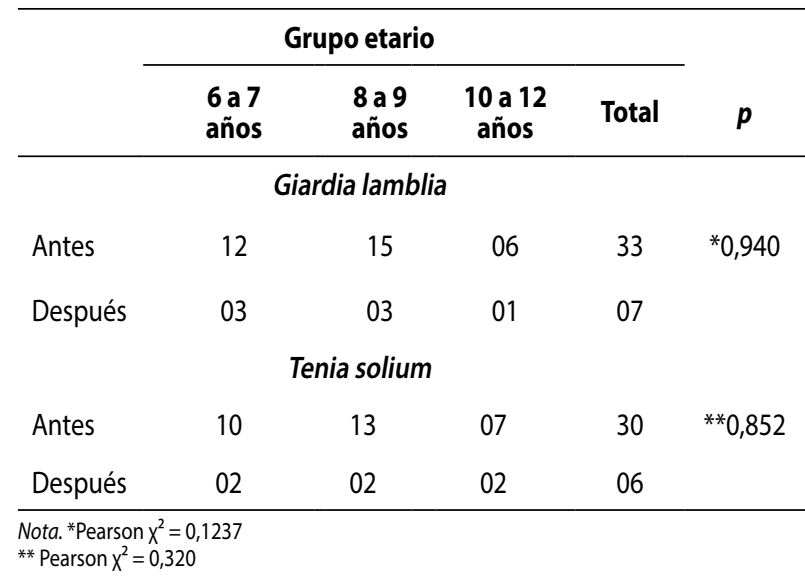

exposición al extracto se encontraron 6 casos de parasitosis intestinal. Por lo tanto, hubo diferencia estadísticamente significativa en el efecto antiparasitario $(p<0,05)$.

En la tabla 4 se presenta la parasitosis intestinal por protozoario (Giardia lamblia) antes y después de administrar extracto experimental según grupo etario. Antes de administrar se encontraron en los sujetos de 6 a 7 años, unos 12 casos de parasitosis, después 3 casos; en los escolares de 8 a 9 años 15 casos y después de la exposición 3 casos, y en el grupo etario de 10 a 12 años, 6 casos antes de la administración del extracto experimental y después 1 caso. Por lo tanto, no existe diferencia estadísticamente significativa en el efecto antiparasitario (Giardia lamblia) según grupo etario $(p>0,05)$.

En la tabla 5 se presenta la parasitosis intestinal por helmintos (Giardia lamblia), antes y después de administrar extracto experimental según sexo. Antes de administrar se encontraron en los escolares varones 20 casos de parasitosis, después 5 casos; en las escolares 13 casos y después de la exposición 2 casos. No existe diferencia estadísticamente significativa en el efecto antiparasitario (Giardia lamblia) según sexo ( $p>0,05)$. En la parasitosis intestinal por helmintos (Tenia solium), antes de administrar se encontraron en los escolares varones 18 casos de parasitosis, después 4 casos; en las escolares 12 casos y después de la exposición 2 casos. Por lo tanto, no existe diferencia estadísticamente significativa en el efecto antiparasitario (Tenia solium) según sexo $p>0,05$.

\section{DISCUSIÓN}

El presente estudio sugiere que el consumo de Allium sativum (ajo), Citrus limon (limón) y Carica papaya (papaya), suministradas como extracto a los escolares durante 5
Tabla 5. Parasitosis intestinal (Giardia lamblia + Tenia solium), antes y después de administrar extracto experimental según sexo

\begin{tabular}{|c|c|c|c|c|}
\hline & \multicolumn{3}{|c|}{ Sexo } & \multirow[b]{2}{*}{$p^{*}$} \\
\hline & Femenino & Masculino & Total & \\
\hline \multicolumn{5}{|c|}{ Giardia lamblia } \\
\hline Antes & 13 & 20 & 33 & 0,591 \\
\hline \multirow[t]{2}{*}{ Después } & 02 & 05 & 07 & \\
\hline & & nia solium & & $P^{* *}$ \\
\hline Antes & 12 & 18 & 30 & 0,760 \\
\hline Después & 02 & 04 & 06 & \\
\hline
\end{tabular}

Nota. ${ }^{*}$ Pearson $x^{2}=0,288$

${ }^{* *} X^{2}=0,760$

días, resultó en una reducción significativa de la infección por parasitosis intestinal (Giardia lamblia y Tenia solium). La tasa general de reducción de parasitosis intestinal por protozoario con el extracto experimental fue de $78,8 \%$ y $80,0 \%$; resultados similares encontrados por Kugo et al., para quienes el consumo de papilla de harina de maíz fortificada con semillas molidas de Carica papaya durante dos meses mostró una reducción en la infección por Ascaris lumbricoides. La tasa general de reducción de huevos del tratamiento con papilla fortificada y albendazol fue del $63,9 \%$ y $78,8 \%$, respectivamente ${ }^{(12)}$. De igual manera, Adbel et al., en su estudio efectos antiprotozoarios in vivo de extractos de ajo (Allium sativum) y jengibre (Zingiber officinale) en ratones infectados experimentalmente con Blastocystis spp, reportaron que el uso de jengibre y ajo para el tratamiento de la blastocistosis es beneficioso ${ }^{(13)}$. También coincide con lo hallado por Avaz et al., quienes evaluaron la actividad antihelmíntica del ajo (Allium sativum) en ratones infectados naturalmente con Aspiculuris tetráptera, y donde encontraron que el ajo fue eficaz durante la duración del tratamiento en ratones ${ }^{(14)}$. Nuestro estudio difiere, no obstante, con los resultados obtenidos por Fallahi et al., quienes demostraron que sativum (ajo) tuvo el menor efecto de letalidad en quistes de $G$. lamblia in vitro $(22,65 \pm 10,47 \%)^{(15)}$. Este débil efecto se puede atribuir a los diferentes métodos utilizados para preparar y procesar el extracto, el efecto de las condiciones ambientales sobre el compuesto del extracto de Allium sativum, hasta el momento de consumir tiempo y diversas especies de Allium sativum en otros estudios.

Además, existen estudios que evidencian el efecto beneficioso del Citrus limon; Shija et al, encontraron en su estudio una supresión máxima del crecimiento del parásito a las 72 h después del tratamiento ${ }^{(16)}$.

Los efectos antihelmínticos del ajo (Allium sativum) son atribuidos a la abundancia de compuestos que contienen 
azufre antiparasitario $^{(17)}$, regula al alza la producción de óxido nítrico en las plaquetas sanguíneas y los macrófagos, que destruyen el parásito ${ }^{(18)}$. El ajo también contiene lectinas de unión a manosa ${ }^{(19)}$ que facilitan la unión del parásito al receptor de la superficie del macrófago, permitiendo que el macrófago engulla al parásito ${ }^{(20)}$. Además, el ajo contiene una fracción inmunomoduladora que cambia el patrón de citocinas de las respuestas inmunitarias mediadas por linfocitos T helper 2 (Th2), responsables de la formación de granulomas a las respuestas inmunitarias mediadas por linfocitos $\mathrm{TH} 1$ responsables de la resistencia inmunitaria ${ }^{(21)}$. También el ajo muestra actividad inmunomoduladora como alteración de la producción de citocinas, secreción de anticuerpos, proliferación de linfocitos, activación de $\mathrm{MQ}$ y CD y promoción de fagocitosis ${ }^{(20)}$.

El limón en su composición química presenta flavonoides a quienes se les atribuye la acción farmacológica ${ }^{(22)}$. Se ha demostrado científicamente que los componentes de la fruta tropical Carica papaya (papaya) tienen potentes propiedades antihelmínticas y antiamoebias ${ }^{(23)}$. Los análisis fitoquímicos de seguimiento muestran que el isotiocianato de bencilo es el ingrediente antihelmíntico bioactivo en las semillas ${ }^{(10)}$. La composición del extracto de la Carica papaya estudios recientes han demostrado la actividad antiprotozoaria de los ácidos grasos con derivados de cadena media (TyC8, TyC10 y TyC12) que exhiben buenas actividades antiparasitarias ${ }^{(24)}$.

Okeniyi, en su investigación clínica aleatorizado mostró que los niños que recibieron $1 \mathrm{~g}$ de dosis de semillas de papaya emulsionadas en casa mostraron una tasa de eliminación de parásitos del $77 \%$ después de siete días en comparación con una tasa de eliminación del $17 \%$ en aquellos que recibieron miel sola ${ }^{(25)}$. En nuestro estudio, el extracto experimental combinado contiene flor de Carica de papaya y mostró reducción significativa de parasitismo infantil.

Debido a los resultados beneficiosos obtenidos de la medicina tradicional en el tratamiento de parasitosis surgió el interés por los fármacos de origen vegetal para el tratamiento de enfermedades parasitarias, además de los graves efectos secundarios provocados por el abuso y mal uso de las drogas sintéticas y la dificultad para acceder al tratamiento farmacológico ${ }^{(26,27)}$.

Las consecuencias de presentar parasitosis intestinal se asocian comúnmente con la desnutrición, anemia, deterioro del crecimiento, escasa asistencia a la escuela y deterioro de la cognición ${ }^{(28-31)}$.
Los resultados de este estudio estuvieron limitados por su pequeño número de pacientes y su naturaleza cuasiexperimental, ya que carece de grupo control positivo o negativo. También la posible limitación del estudio incluye la no aleatorización de los grupos de pacientes.

A partir de los resultados generales podemos concluir que el extracto experimental Allium sativum (ajo), Citrus limon (limón) y Carica papaya (papaya) presentó diferencias significativas antes y después de ser administrado frente a la parasitosis intestinal. El ajo, limón y flor de papaya pueden ser útiles como tratamiento alternativo contra la parasitosis intestinal en humanos.

\section{Agradecimiento}

A los profesionales del área de laboratorio clínico del Hospital Regional Hermilio Valdizán de Huánuco, quienes procesaron la muestra para el examen parasitológico.

\section{REFERENCIAS}

1. Baker C. Red boom: Atlas de enfermedades infecciosas en pediatría. Buenos Aires: Editorial Médica Panamericana; 2009.

2. Jiménez J, Vergel K, Velásquez $M$, Vega $F$, Asceta $R$, Romero $S$, et al. Parasitosis en niños en edad escolar: relación con el grado de nutrición y aprendizaje. Rev Hor Mes. [Internet] 2011; 11(2): 65-69 [Consultado 2020 Abr 22]; Disponible en: https://medicina.usmp.edu.pe/ medicina/horizonte/2011_2/Art1_Vol11_N2.pdf

3. Juárez M, Rajal V. Parasitosis intestinales en Argentina: principales agentes causales encontrados en la población y en el ambiente. Rev Argent Microbiol. [Internet] 2013; 45(3): 191-204 [Consultado 2020 May 12] Disponible en: https://pubmed.ncbi.nlm.nih. gov/24165144/

4. Naquira C. Taenia solium: biological cycle and characteristics. En: García HH, Martínez SM: Taenia solium Taeniasis / Cysticercosis. 2a ed. Lima: Editorial Universo; 1999.

5. Maco V, Raymundo L, Terashima A, Samalvides F, Gotuzzo E. Distribución de la enteroparasitosis en el altiplano peruano: estudio en 6 comunidades rurales del departamento de Puno, Perú. Rev. Gastroenterol. [Internet] 2002; 22(4): 304-309 [Consultado 2020 May 12] Disponible en: http:// www.scielo.org.pe/scielo.php?script $=$ sci_ arttext\&pid=S1022-51292002000400006

6. Pajuelo G, Lujan D, Paredes B. Estudio de enteroparásitos en el hospital de Emergencias Pediátricas, Lima-Perú. Rev Med Hered. [Internet] 2005; 16 (3): 178-183 [Consultado 2020 May 15] Disponible en: http://www.scielo.org.pe/ $\mathrm{pdf} / \mathrm{rmh} / \mathrm{v} 16 \mathrm{n3} / \mathrm{v} 16 \mathrm{n} 3$ ao3.pdf

7. Asadi-Samani M, KootiW, Aslani E, Shirzad H. Una revisión sistemática de las plantas medicinales de Irán con efectos contra el cáncer. Alternativa complementaria. J. Evid Med. [Internet] 2016; 21(2): 143-153 [Consultado 2020 May 15] Disponible en: https://journals.sagepub. com/doi/pdf/10.1177/2156587215600873 
8. Yousefi E, Eskandari A, Gharavi MJ, Khademvatan S. In vitro activity and cytotoxicity of Crocus sativus extract against leihmania major (MRHO/IR/75/ER). Infect Disord Drug Targets. [Internet] 2014; 14 (1): 56-60 [Consultado 2020 May 15] Disponible en: https://pubmed.ncbi.nlm. nih.gov/25159304/

9. Abou O, Basyoni M, Mahdy O, Bocktor N. El efecto de las semillas de preziquantel y Carica papaya sobre la infección por hymenolepis nana en ratones utilizando un microscopio electrónico de barrido. Parasitol Res. [Internet] 2014; 113: 2827-2836 [Consultado 2020 May 15] Disponible en: https://pubmed.ncbi.nlm.nih. gov/24849866/

10. Kermanshai R, McCarry B, Rosenfeld J, Summers P, Weretilnyk $E$, Sorger $G$. El isotiocianato de bencilo es el principal o único antihelmíntico en los extractos de semillas de papaya. Fitoquímica. [Internet] 2001; 57: 427-435 [Consultado 2020 May 15] Disponible en: https://www.sciencedirect.com/science/article/abs/pii/ S0031942201000772?via\%3Dihub.

11. Haftu D, Deyessa N, Agedew E. Prevalencia y factores determinantes de los parásitos intestinales entre los escolares de la ciudad de Arba Minch, en el sur de Etiopía. Am J Health Res. [Internet] 2014; 2(5): 247-254 [Consultado 2020 May 15] Disponible en: http://www. sciencepublishinggroup.com/journal/paperinfo?journalid=221\&doi=10.11648/j.ijbmr.20190701.11

12. Kugo M, Keter L, Maiyo A, Kinyua J, Ndemwa P, Maina G, et al. Fortification of Carica papaya fruit seeds to school meal snacks may aid Africa mass deworming programs: a preliminary survey. BMC Complement Altern Med. [Internet] 2018; 18(1): 327 [Consultado 2020 Jul 2] Disponible en: https://www.ncbi.nlm.nih.gov/pmc/articles/ PMC6286506/pdf/12906_2018_Article_2379.pdf

13. Abdel-Hafeez E, Ahmad A, Kamal A, Abdellatif M, AbdeIgelil N. In vivo antiprotozoan effects of garlic (Allium sativum) and ginger (Zingiber officinale) extracts on experimentally infected mice with Blastocystis spp. Parasitol Res. [Internet] 2015; 114 (9): 3439-44 [Consultado 2020 Jul 5] Disponible en: https://link.springer.com/article/10.1007\%2Fs00436-015-4569-x

14. Ayaz E, Türel I, Gül A, Yilmaz O. Evaluation of the anthelmentic activity of garlic (Allium sativum) in mice naturally infected with Aspiculuris tetraptera. Recent Pat Antiinfect Drug Discov. [Internet] 2008 Jun; 3(2): 149-52 [Consultado 2020 Jul 14] Disponible en: https://pubmed.ncbi.nlm.nih.gov/18673129/

15. Fallahi S, Rostami A, Delfan B, Pournia Y, Rashidipour M. Effect of olive leaf, Satureja khuzestanica, and Allium sativum extracts on Giardia lamblia cysts compared with metronidazole in vitro. J Parasit Dis. [Internet] 2016; 40(4): 1204-1209 [Consultado 2020 Jul 14] Disponible en: https://www.ncbi.nlm.nih.gov/pmc/articles/ PMC5118276/pdf/12639_2015_Article_650.pdf

16. Shija K, Nondo R, Mloka D, Sangeda R, Bwire G. Effects of lemon decoction on malaria parasite clearance and selected hematological parameters in Plasmodium berghei ANKA infected mice. BMC Complement Med Ther. [Internet] 2020 Ene 30; 20(1): 24 [Consultado 2020 Jul 21] Disponible en: https://pubmed.ncbi.nlm.nih. gov/32020885/

17. Zenner L, Callait M, Granier C, Chauve C. Efecto in vitro de los aceites esenciales de Cinnamomum aroma- ticum, Citrus limon y Allium sativum en dos flagelados intestinales de aves de corral, Tetratrichomonas gallinarum e Histomonas meleagridis. Parásito [Internet] 2003; 10(2): 153-157 [Consultado 2020 Jul 3] Disponible en: https://www.parasite-journal.org/articles/parasite/ pdf/2003/02/parasite2003102p153.pdf

18. Andrade Z. Regresión de esquistosomiasis y fibrosis hepática. Acta Trop. [Internet] 2008; 108(2): 7982 [Consultado 2020 Ago 1] Disponible en https:// www.sciencedirect.com/science/article/abs/pii/ S0001706X08000831?via\%3Dihub

19. Das I, Hirani J, Sooranna S. La arginina no es responsable de la activación del óxido nítrico sintasa por el ajo. J Ethnopharmacol. [Internet] 1996; 53(1): 5-9 [Consultado 2020 Ago 4] Disponible en: https://pubmed.ncbi.nlm. nih.gov/8807470/

20. Dam T, Bachhawat K, Rani P, Surolia A. Las lectinas de ajo (Allium sativum) se unen a cadenas de oligosacáridos con alto contenido de manosa. J Biol Chem. [Internet] 1998; 273(10): 5528-35 [Consultado 2020 Ago 3] Disponible en: https://www.jbc.org/action/showPdf?pi$\mathrm{i}=$ S0021-9258\%2818\%2967805-6

21. Gamboa M, Aranda I, Mut-Martín M. Control in vivo e in vitro de Leishmania mexicana debido a la producción de NO inducida por el ajo. Scand J Immunol. [Internet] 2007; 66: 508-514 [Consultado 2020 Ago 3] Disponible en: https://onlinelibrary.wiley.com/doi/epdf/10.1111/j.1 365-3083.2007.02000.x

22. Del Río J, Fuster M, Gómez P, Porras I, García A., Ortuño A. Citrus limón: Una fuente de flavonoides de interés farmacéutico. Química de los alimentos. [Internet] 2004; 84(3): 457-461 [Consultado 2020 Ago 5] Disponible en: https://www.sciencedirect.com/science/article/abs/pii/ S0308814603002723?via\%3Dihub

23. Abou O, Basyoni M, Mahdy O, Bocktor N. El efecto de las semillas de preziquantel y Carica papaya sobre la infección por hymenolepis nana en ratones utilizando un microscopio electrónico de barrido. Parasitol Res. [Internet] 2014; 113(8): 2827-2836 [Consultado 2020 Ago 19] Disponible en: https://pubmed.ncbi.nlm.nih. gov/24849866/

24. Aissa I, Sghair R, Bouaziz M, Laouini D, Sayadi S, Gargouri Y. Síntesis de derivados de ésteres de tirosilo lipófilos y evaluación de sus actividades antimicrobianas y antileishmania. Lípidos Salud Dis. [Internet] 2012; 11:13 [Consultado 2020 Ago 19] Disponible en: https://www. ncbi.nlm.nih.gov/pmc/articles/PMC6270640/pdf/molecules-18-12621.pdf

25. Okeniyi J, Ogunlensi A, Oyelami A, Adeyemi A. Eficacia de las semillas secas de Carica papaya contra la parasitosis intestinal humana: un estudio piloto. J Med Food. [Internet] 2007; 10(1): 194-196 [Consultado 2020 Abr 22] Disponible en: https://pubmed.ncbi.nlm.nih. gov/17472487/

26. Tarifas S. Plantas como fuente de drogas. Toxicon. [Internet] 2001; 39: 603-613 [Consultado 2020 May 5] Disponible en: http://www.farmacognosia.ufpr.br/pdf/ rates_plant.pdf

27. Martins G, Silva R, Araújo E, Pereira M, Vieira $H$, Viana A. Influencia del tipo de fruto, peso específico de la semilla y período de almacenamiento en la calidad fisiológica de las semillas de papaya del grupo de las formosas. Rev Bras Sementes. [Internet] 2005; 27: 12-17 
[Consultado 2020 Jul 2] Disponible en: https://www. scielo.br/pdf/rbs/v27n2/a03v27n2.pdf

28. Hall A, Hewitt G, Tuffrey V, de Silva N. Una revisión y metanálisis del impacto de las lombrices intestinales en el crecimiento y la nutrición infantil. Matern Child Nutr. [Internet] 2008; 4(1): 118-236 [Consultado 2020 Ago 22] Disponible en: https://www.ncbi.nlm.nih.gov/pmc/articles/PMC6860651/

29. Albonico M, Allen H, Chitsulo L, Engels D, Gabrielli AF, Savioli L. Control de la helmintiasis transmitida por el suelo en niños en edad preescolar mediante quimioterapia preventiva. PLoS Negl Trop Dis. [Internet] 2008; 2(3): 126 [Consultado 2020 Set 23] Disponible en: https://
www.ncbi.nlm.nih.gov/pmc/articles/PMC2274864/pdf/ pntd.0000126.pdf

30. Le H, Brouwer I, Verhoef H, Nguyen K, Kok F. Anemia e infección por parásitos intestinales en escolares de zonas rurales de Vietnam. Asia Pac J Clin Nutr. [Internet] 2007; 16(4): 716-723 [Consultado 2020 Set 23] Disponible en: http://apjcn.nhri.org.tw/server/APJCN/16/4/716.pdf

31. Oberhelman R, Guerrero E, Fernández $M$, Silio M, Mercado $\mathrm{D}$, Comiskey N, et al. Correlaciones entre parasitosis intestinal, crecimiento físico y desarrollo psicomotor en lactantes y niños de zonas rurales de Nicaragua. Am J Trop Med Hyg. [Internet] 1998; 58(4): 470-475 [Consultado 2020 Set 23] Disponible en: https://www.ajtmh.org/view/journals/ tpmd/58/4/article-p470.xml?tab_body=pdf 